\title{
Methods for Specular Color Component Accelerate Calculation
}

\author{
Olexandr Romanuyk ${ }^{1}$, Anatolij Chernij ${ }^{2}$ \\ 1) Ph.d, Vinnitsa national technical university, Ukraine, Vinnitsa, Ivana Boguna str, 119, \\ E-mail ran@cec.vstu.vinnica.ua \\ 2) Ph.d, Vinnitsa national technical university, Ukraine, Vinnitsa, Ivana Bevza str, 16/20
}

\begin{abstract}
The new approaching for Phong shading method speed increasing is presented in given paper. According to the first approach for those parts of image were highlights is presented is used the Blinn lightning model and for the rest part the simple Lambert model is applied. Using this method the quality of scene stay the same when we use the complicated Blinn model and the scene creation speed is razed significantly. For Phong shading method is proposed the approach accordingly to which the normal for the current pixel in triangle can be calculated using the normals to the two privies pixels. Given approaches can significantly decrease the amount of calculation and thus increase the shading speed.
\end{abstract}

Keywords - rendering, shading, lighting models

\section{INTRODUCTION}

During realistic image forming the most timeconsuming procedure is the shading. The existing shading procedure are based in the fact that almost all surfaces can be presented be polygonal meshes. If for all meshes is used one normal during shading procedure we get the images which are consists of separate polygons with strictly visually noticeable colour intensity changing in borders.

Gouraud [1] proposed method how to get more realistic images. He proposed to approximate the normals in the polygon vertexes. The colours intensity are calculated for each vertexes and then linearly interpolated across polygon edges and raster lines. Gouraud method is good only to generate the mat surface, because linear interpolation don't take in to account the surface curvature.

The best quality of shading can be achieved using the method which was proposed by Phong.[2]. During the Phong shading are taking in to account normals to all polygons pixels. The calculated normals and information about observer and light source location are used for colour intensity calculation.

In Phong method during normals calculation it is necessary to do their normalization. This operation is a quite time consuming. To avoid this operation Shoemake [3] proposed to use for normals calculation instead of linear interpolation angular interpolation (fig.1). In this case normal can be calculated using the formula:

$$
\vec{N}(w)=\vec{N}_{a} \frac{\sin ((1-w) \psi)}{\sin \psi}+\vec{N}_{b} \frac{\sin (w \psi)}{\sin \psi},
$$

were $w \in[0,1]$, and $\psi-$ the angle between normals $\vec{N}_{a}$ and $\vec{N}_{b}$.

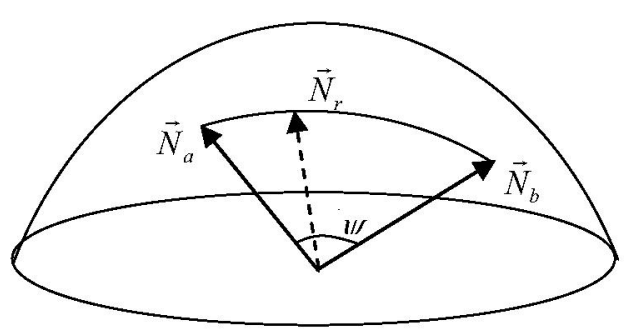

Fig. 1. Angular interpolation.

Nevertheless equation (1) is still a quite complicated. In given paper we present the new approach to simplify normals calculation during Phong shading using angular interpolation.

When normals are founded they are used to calculate colour intensity. For this purpose are used lighting models. The most common used lighting models are Lambert models [4] - for mat surfaces and Phong [2], Blinn models [5] - for surface which produce highlights .

The basic purpose of Blinn and Phong lightning models usage is to show the increased colour intensity zone (highlight) on a surface. The highlight zone is observed only on the limited part of object. Its accommodation depends on orientation of object in space, place of the observer presence and external light source, and the sizes depends on object specular characteristics, which are determined by coefficient $n$. The large values of $n$ gives the focused distribution of highlight, and small - wider distribution.

Usually as lightning model often Blinn model is used. At identical results of use the given model differs considerably by smaller computing complexity in comparison with Phong model. The formula, according to which the intensity of color is calculated has the 
following kind:

$$
\begin{gathered}
I_{B}=I_{p}(\lambda) \cdot k_{p}(\lambda)+I_{l}^{i n}(\lambda) \cdot k_{d}(\lambda) \cdot(\vec{N} \cdot \vec{L})_{+} \\
+I_{l}^{i n}(\lambda) \cdot k_{s p}(\lambda) \cdot \cos ^{n} \gamma,
\end{gathered}
$$

where $I_{p}(\lambda), I_{l}^{\text {in }}(\lambda)$ - the intensity of ambient and external light source accordingly, $\vec{N}$ - normal to the surface, $\vec{L}$ - vector of a light direction , $\gamma$ - angle between normal $\vec{N}$ and vector $\vec{H}, \vec{H}$ is calculated according to the formula $(\vec{L}+\vec{V}) /|\vec{L}+\vec{V}|$, where $\vec{V}$ vector of observer location, $k_{p}(\lambda), k_{d}(\lambda), k_{s p}(\lambda)$ factors of ambient, diffuse and specular lights, $n$ - factor of surface secularity.

The formula for colour intensity calculation in Lambert lightning model [4] is identical to the formula (2) with that difference, that $I_{l}^{\text {in }}(\lambda) \cdot k_{s p}(\lambda) \cdot \cos ^{n} \gamma$, which responds for highlight formation on a surface is equaled to zero:

$$
I_{L}=I_{p}(\lambda) \cdot k_{p}(\lambda)+I_{l}^{\text {in }}(\lambda) \cdot k_{d}(\lambda) \cdot(\vec{N} \cdot \vec{L}) .
$$

Lambert lightning model does not allow to form highlight on a surface and consequently is suitable only for lustreless images formation.

Because of $I_{l}^{i n}(\lambda) \cdot k_{s p}(\lambda) \cdot \cos ^{n} \gamma \quad$ absence, the Lambert lightning model computing complexity is less for Blinn lightning model computing complexity.

The new method is presented, according to which, for those parts of object, where the highlight zone is observed, it is necessary to use Blinn lightning model and for all other parts of object - simple Lambert lightning model. The quality remains the same, as at use of Blinn lightning model and because the specular component $I_{l}^{\text {in }}(\lambda) \cdot k_{s p}(\lambda) \cdot \cos ^{n} \gamma$ is computed only in case of necessity, the speed of shading process raises considerably.

\section{ANGULAR INTERPOLATION}

Lets rewrite the equation (1), which was proposed by Shoemake for normals culculation using trigonometric formula:

$$
\vec{N}(w)=\vec{N}_{a} \frac{\sin \psi \cos (w \cdot \psi)-\cos \psi \sin (w \cdot \psi)}{\sin \psi}+\vec{N}_{b} \frac{\sin (w \cdot \psi)}{\sin \psi} .
$$

After simplification:

$\vec{N}(w)=\vec{N}_{a} \cos (w \cdot \psi)-\vec{N}_{a} \frac{\cos \psi \sin (w \psi)}{\sin \psi}+\vec{N}_{b} \frac{\sin (w \cdot \psi)}{\sin \psi}$.

Last eqution can be rewritten in the next form:

$$
\vec{N}(w)=\vec{N}_{a} \cos (w \cdot \psi)+\frac{\vec{N}_{b}-\vec{N}_{a} \cdot \cos \psi}{\sin \psi} \sin (w \cdot \psi) .
$$

If to change in folmula (4) $\sin \psi$ function to $\sqrt{1-\cos ^{2} \psi}$, and present $\cos \psi$ like a scalar product of $\vec{N}_{a}$ and $\vec{N}_{b}$ will get:

$\vec{N}(w)=\vec{N}_{a} \cos (w \cdot \psi)+\frac{\vec{N}_{b}-\vec{N}_{a} \cdot\left(\vec{N}_{b} \bullet \vec{N}_{a}\right)}{\sqrt{1-\left(\vec{N}_{b} \cdot \vec{N}_{a}\right)^{2}}} \sin (w \cdot \psi) \cdot($

Multiplier $\left(\vec{N}_{b}-\vec{N}_{a} \cdot\left(\vec{N}_{b} \bullet \vec{N}_{a}\right)\right) / \sqrt{1-\left(\vec{N}_{b} \cdot \vec{N}_{a}\right)^{2}}$ is a vector $\vec{N}_{k}$ (fig. 2).

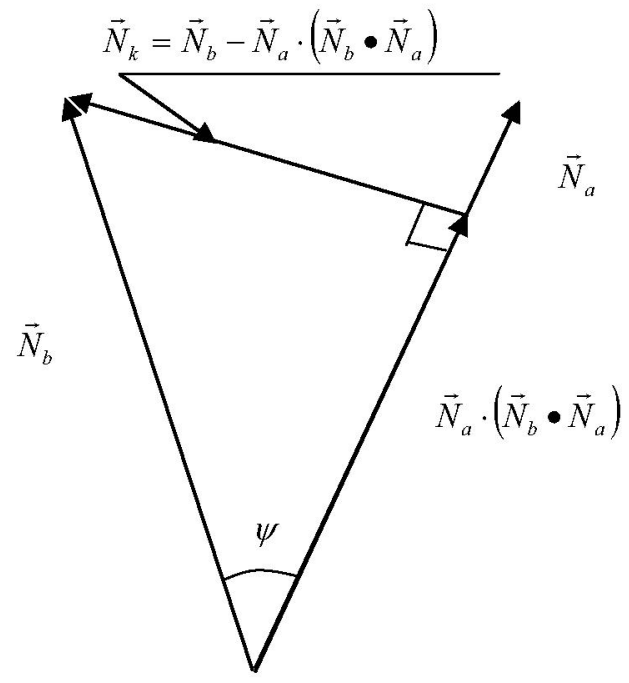

Fig. 2. Vector $\vec{N}_{k}$ calculation.

And equation (5) can be rewritten in next way:

$$
\vec{N}(w)=\vec{N}_{a} \cos (w \cdot \psi)+\vec{N}_{k} \sin (w \cdot \psi),
$$

If raster line have $m$ pixels, angle bettwen two neighbor normals be:

$$
\varphi=\frac{\psi}{m},
$$

were $\psi=\arccos \left(\vec{N}_{a} \bullet \vec{N}_{b}\right)$.

Using this remark the equation (6) can be rewritten:

$$
\vec{N}(t)=\vec{N}_{a} \cos (t \cdot \varphi)+\vec{N}_{k} \sin (t \cdot \varphi),
$$

were $t$ - pixel number along raster line, $t \in[0, m]$.

Lets write the equation (7) in iteration form. Find $\vec{N}(t+1)$ and $\vec{N}(t-1)$.

$$
\begin{gathered}
\vec{N}(t+1)=\vec{N}_{a} \cos ((t+1) \cdot \varphi)+\vec{N}_{k} \sin ((t+1) \cdot \varphi)= \\
\vec{N}_{a} \cdot \cos (t \varphi) \cdot \cos \varphi-\vec{N}_{a} \cdot \sin (t \varphi) \cdot \sin \varphi+ \\
\quad+\vec{N}_{k} \cdot \sin (t \varphi) \cdot \cos \varphi+\vec{N}_{k} \cdot \cos (t \varphi) \cdot \sin \varphi
\end{gathered}
$$




$$
\begin{gathered}
\vec{N}(t-1)=\vec{N}_{a} \cos ((t-1) \cdot \varphi)+\vec{N}_{k} \sin ((t-1) \cdot \varphi)= \\
\quad \vec{N}_{a} \cdot \cos (t \varphi) \cdot \cos \varphi+\vec{N}_{a} \cdot \sin (t \varphi) \cdot \sin \varphi+ \\
\quad+\vec{N}_{k} \cdot \sin (t \varphi) \cdot \cos \varphi-\vec{N}_{k} \cdot \cos (t \varphi) \cdot \sin \varphi
\end{gathered} .
$$

Adding $\vec{N}(t+1)$ and $\vec{N}(t-1)$ we will get

$\vec{N}(t+1)+\vec{N}(t-1)=2 \cdot \cos \varphi \cdot\left(\vec{N}_{a} \cos (t \cdot \varphi)+\vec{N}_{k} \sin (t \cdot \varphi)\right.$.

Using formula (7) the last equation can be written in next form:

$$
\vec{N}(t+1)+\vec{N}(t-1)=2 \cdot \vec{N}(t) \cdot \cos \varphi .
$$

From here

$$
\vec{N}(t+1)=2 \cdot \vec{N}(t) \cdot \cos \varphi-\vec{N}(t-1) .
$$

We show have the unit normal using angular interpolstion can be founded from two privious.

If take in to account, that $w \cdot \psi$ can be founded using cumulative summation, for calculation of normal ortogonal component using formula (1) it is necessary to do 2 division, 2 multiplication, 3 addition 2 operetion of cine calculation. Such operetion have to be done for three vector components. Using formula (8) it have to be done only 1 multiplication, 1 substruction and 1 shaiftion for each vector components calculation.

\section{ADAPTIVE SHADING}

How it was shown in introduction it is have a sense to use the complicated lighting model only if highlight zone is presented in surface in other case can be used simple model. This conception was put in basic in developing adaptive shading method.

The basic idea of a given method is to enter boundary value of zero for function $\cos ^{n} \gamma$. At enough large $n$, the function $\cos ^{n} \gamma$ quickly achieves practically zero value, from which the further calculation becomes inexpedient. For boundary value of zero it is offered to take the size $2^{-q}$, where $q$ gets out depending on necessary accuracy of colour intensity definition. We make the appropriate equation

$$
\cos ^{n} \gamma_{n}=2^{-q},
$$

where $\gamma_{n}-$ angle, at which the function $\cos ^{n} \gamma_{n}$ accepts the zero value.

Taking into account equation (9) we calculate angle $\gamma_{n}$ value: $\gamma_{n}=\arccos \left(2^{\left(-\frac{q}{n}\right)}\right)$.

The size $q$ computation is offered to do in a way than difference between real zero value of specular color intensity component $I_{l}^{i n}(\lambda) \cdot k_{s p}(\lambda) \cdot \cos ^{n} \gamma$ and received as a result of zero threshold value usage for function $\cos ^{n} \gamma$, was in borders from zero to one:

$$
0 \leq I_{i}^{6 x}(\lambda) \cdot k_{s p}(\lambda) \cdot \cos ^{n} \gamma_{n} \leq 1 .
$$

Having replaced value of function $\cos ^{n} \gamma$ on size $2^{-q}$ we will receive

$$
0 \leq I_{l}^{i n}(\lambda) \cdot k_{s p}(\lambda) \cdot 2^{-q} \leq 1 .
$$

The value of $k_{s p}(\lambda)$ is in a range $[0,1]$, whence maximal value of factor $k_{s p}(\lambda)=1$.

The value $I_{i}^{\text {in }}(\lambda)$ can vary from zero to infinity, depending on how powerful the light source is. For convenience the given size is offered to be presented by the nearest number, which is a degree of two as follows: $I_{l}^{\text {in }}(\lambda) \leq 2^{t}$. Substituting unknown sizes in the equation (10) receive, that $q=t$.

In practice at realistic images formation, as a rule, intensity of a light source does not exceed 500 .

Substituting the given size in the equation (10) we will get $q \approx 9$.

Thus angle threshold value $\gamma_{n}$, after which there is no necessity to calculate specular component ${ }_{l}^{i n}(\lambda) \cdot k_{s p}(\lambda) \cdot \cos ^{n} \gamma$, is

$$
\gamma_{n}=\arccos \left(2^{\left(-\frac{9}{n}\right)}\right) \Rightarrow \cos \gamma_{n}=2^{\left(\frac{-9}{n}\right)} .
$$

The researches necessity of above mentioned lightning models usage is offered to be carried out not for each pixel separately, and for groups of pixels. The basic figure, which is shading is the triangle. Accordingly the researches will be carried out for pixels groups limited by a triangle.

All triangles are divided on two types:

1) triangles, where the highlight or part of it is observed;

2) triangles, where the highlight zone is not observed.

The triangles can be carried out to the first group, if for all pixels or part them, the angle $\gamma$ is in a range:

$$
0 \leq \gamma \leq \arccos \left(2^{\left(-\frac{9}{n}\right)}\right) .
$$

For shading of such triangles the Blinn lightning model will be used.

To the second group concern a triangle, at which the size of a angle $\gamma$ for all or some part of pixels is more 
for size $\arccos 2^{\left(-\frac{9}{n}\right)}$ and for such triangles will be used simple Lambert model.

There is no sense to analyze size of a angle $\gamma$ for each of pixels of a triangle, it is enough to carry out such analysis only for pixels, which are placed on triangle edges.

All possible variants of middle way vector accommodation concerning to normal vectors of edge pixels in triangle is possible to divide in two groups:

a) The middle way vector is placed in such a manner that has the least angle $\gamma$ with normal vector for one of the last edge pixels.

в) The middle way vector is placed in such a manner that has the least angle $\gamma$ with normal vector for one of pixels witch is situated in edge middle.

For definition of the fact to which of variants of middle way vector accommodation can be carried out the triangle edge is offered to use spherical trigonometry.

In a fig. 3 the spherical triangle is submitted, in which basis make two normal vectors $\vec{N}_{A}, \vec{N}_{B}$ of edge AB final points and vector $\vec{H}$.

In case one of angles $\vartheta, v$ is more then $90^{\circ}$, such edge concerns to group a), if both angles $\vartheta, v$ are less $90^{\circ}$, such edge concerns to group в).

Using properties of a spherical triangle receive, that

$$
\begin{aligned}
& \cos \vartheta=(\cos \alpha-\cos \chi \cos \psi) / \sin \psi \sin \chi . \\
& \cos v=(\cos \chi-\cos \alpha \cos \chi) / \sin \psi \sin \alpha .
\end{aligned}
$$

At positive values of $\cos \vartheta$ and $\cos v,<v$ and $<\vartheta$ less $90^{\circ}$, and at negative - is more.

The account of the minimal value of a angle $\gamma$ is made for group a) as follows.

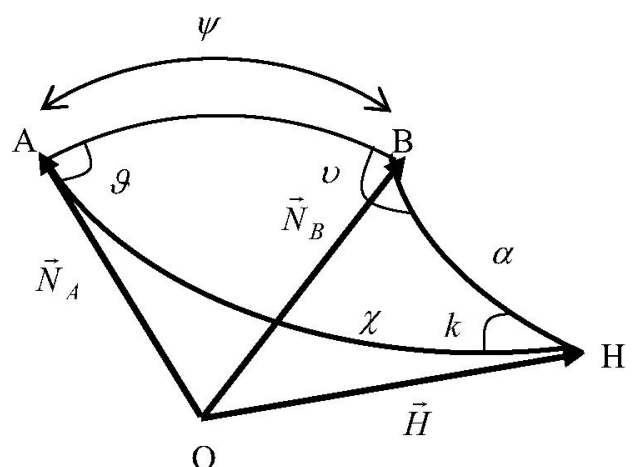

Fig. 3. Spherical triangle in which basis

$$
\text { lays vectors } \vec{N}_{A}, \vec{N}_{B} \text { and } \vec{H} \text {. }
$$

From two angles $<v$ and $<\vartheta$ (fig. 3) is fined out the greatest by comparison cosine marks of the appropriate angles calculated according to the formulas (11) and (12). If a angle $<v$ greater for a angle $<\vartheta$, there is a size $\cos \gamma$ is calculated:

$$
\cos \gamma=\vec{N}_{A} \cdot \vec{H} .
$$

For a case, when a angle $<\vartheta$ greater for a angle $<v$, the size $\cos \gamma$ is equal to:

$$
\cos \gamma=\vec{N}_{B} \cdot \vec{H} .
$$

The received value $\cos \gamma$ is compared with $\cos \gamma_{n}$, which.

If the edge is corresponded to group в) (fig. 4), the calculation of the minimal angle value will be carried out in the next way.

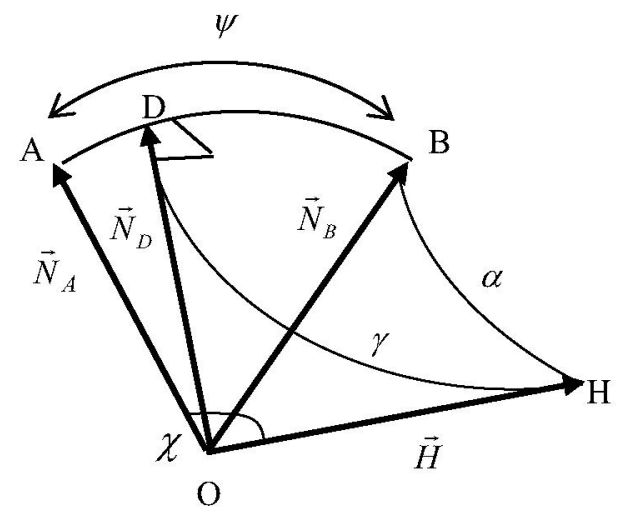

Fig. 4. Angle $\gamma$ cosine calculation.

Let's designate $\vec{N}_{A} \times \vec{N}_{B}$ as a vector $\vec{G}$. From here cosine of angle $\gamma$ is:

$$
\begin{gathered}
\cos \gamma=\sin \left(\frac{\pi}{2}-\gamma\right)=\frac{\vec{G} \times \vec{H}}{\vec{G}}= \\
=\sqrt{\frac{\cos ^{2} \chi-2 \cdot \cos \chi \cdot \cos \psi \cdot \cos \alpha+\cos ^{2} \alpha}{1-\cos ^{2} \psi}} .
\end{gathered}
$$

The received value of size $\cos \gamma$ is compared to size $\cos \gamma_{n}$, which was calculated in formula (15).

Thus, if for each edge of a triangle is satisfied condition, that $\cos \gamma>\cos \gamma_{n}$, in this triangle is not observed highlight zones and it can be shaded with Lambert illumination model usage (equation (3)). In case even for one of triangle edges $\cos \gamma<\cos \gamma_{n}$, then in the given triangle the part of a highlight zone is located and for such triangle it is necessary to use Blinn model (equation (2)) 


\section{CONCLUSION}

Realization of proposed method showed, that objects which were generated using proposed methods are the same to the objects which were shading according to Phong method with use of Blinn model (figure 5).
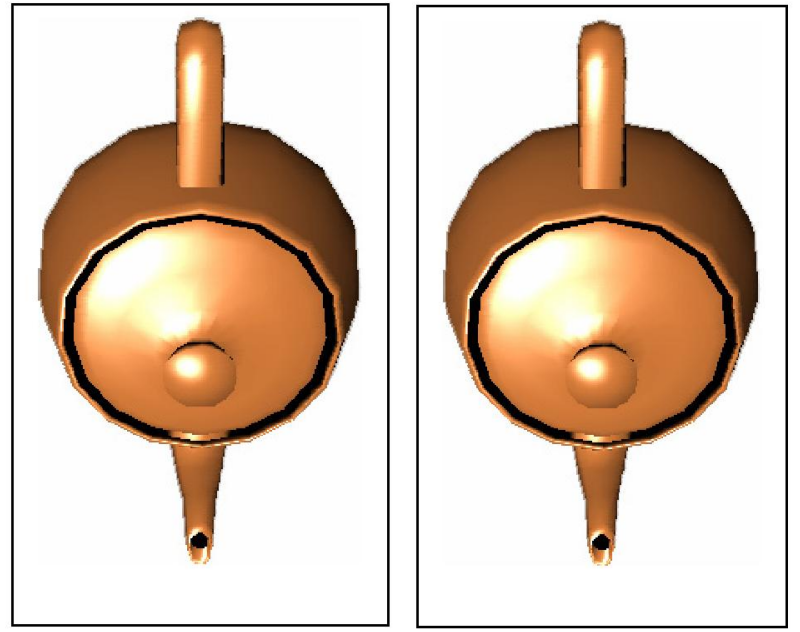

Fig. 5. Example of object Phong shading with Blinn illumination model (left) and with proposed methods (right).
Visual identity is also confirmed by NMSE, which is in range $0 \leq N M S E \leq 0,0001$.

The usage of improved angular interpolation during Phong shading and the method of adaptive approach for objects shading allows essentially lower computing expenses and therefore increase productivity.

Thus the shading quality remains same as well as at use of classical Phong shading method and Blinn lightning model for shading of all triangles, of which the object consists and speed of image generation increase greatly.

\section{REFERENCES}

[1] Gouraud H., "Continuous shading of curved surfaces." IEEE Trans. on Comp," №6, June 1971, pp. 623-628.

[2] Phong B.T., "Lightning for computer generated images," Comm. of the ACM, 18(6), June 1975, pp. 311-317.

[3] Shoemake K. "Animating rotation with quaternion curves," ACM SIGGRAPH, vol. 19. July 1985, pp. 245-254.

[4] Foley, Van Dam, Feiner, and Hughes, Computer Graphics, Principles and Practice, Addison Wesley, 1996, Ch. 16, pp. 800870.

[5] Blinn James F., Models of light reflection for computer synthesized pictures, Computer graphics, vol. 11,1977, pp. 192198. 